\title{
Berry's phase in the two-level model
}

\author{
A.C. Aguiar Pinto, M. Moutinho \\ Curso de Física, Universidade Estadual de Mato Grosso do Sul, Caixa Postal: 351, \\ Cidade Universitária de Dourados, CEP 79804-970, Dourados-MS, Brazil \\ M.T. Thomaz* \\ Instituto de Física, Universidade Federal Fluminense, \\ Av. Gal. Milton Tavares de Souza s/n ${ }^{\circ}$, CEP 24210-346, Niterói-RJ, Brazil
}

(Received on 10 April, 2009)

\begin{abstract}
We study the adiabatic evolution of a two-level model in the presence of an external classical electric field. The coupling between the quantum model and the classical field is taken in the electric dipole approximation. In this regime, we show the absence of geometric phases in the interacting two-level model in the presence of any periodic real time-dependent classical electric field. We obtain a conservative scalar potential in the calculation of Berry's phases of the instantaneous eigenstates of the model. For complex electric fields, we recover the existence of geometric phases. In particular, the geometric phases of the instantaneous eigenstates of the model in the presence of a positive or of a negative frequency component of the monochromatic electric field differ by an overall sign. As a check on our results, we map this interacting two-level model onto a spin- $1 / 2$ model under the action of a classical magnetic field. We confirm that the first one acquires Berry's phase only in the rotating wave approximation [RWA].
\end{abstract}

Keywords: Berry's phase, two-level model

\section{INTRODUCTION}

A wide application of quantum systems with two energy levels can be found in Physics. One important example is the spin- $1 / 2$ model coupled to an external magnetic field, which describes the spin-1/2 magnetic dipole in nuclear magnetic resonance[1, 2]. Another example is the quasi-resonant regime of an atom under the action of a monochromatic laser whose wavelenght is much larger than the average electronic radius. The latter phenomenon is described by the rotating wave approximation (RWA) of the two-level model[3]. In this approximation, the anti-resonant part of the probability transition is neglected, which corresponds to replacing the monochromatic electric field by its positive frequency component.

The two-level models have the nice feature of being exactly soluble and hence good toy models. They can be used to understand the conditions under which a given approximation can be applied. In Berry's original paper about the geometric phases [4], he showed how those phases are acquired by instantaneous eigenstates of the spin-1/2 model adiabatically evolving in the presence of an external periodic classical magnetic field. In Ref.[5] we studied the spin- $1 / 2$ hamiltonian, and derived the exact instantaneous eigenstates of this model in the presence of a precessing classical magnetic field with fixed norm and compared them to the approximate eigenstates obtained from the adiabatic theorem[6, 7].

Since the publication of Ref.[4], discussion on the presence of geometric phases in the two-level system derived from the coupling of matter with a monochromatic electric field [3] can be found in the literature. Its RWA was discussed in Refs. [8, 9]; an irreversible version is presented in Ref. [10]; and a periodically driven two-model with time-dependent diagonal elements can be found in [11]. There has been a recent

${ }^{*}$ Electronic address: mtteif.uff.br revival of the two-level model, as it has been suggested as a prototype for a quantum bit (qubit). In 2003, Leibfired et al. used geometric phases associated to the two-model model, in a quasi-resonant regime, of an atom under the action of a monochromatic laser to model a logic gate[12]. The quantum state has to have a cyclic evolution[13] in the presence of an periodic monochromatic external electric field, that has not adiabatic evolution, since it has to be in the quasi-resonant regime for the two-level model to describe to interaction of matter and radiation.

The aim of the present communication is to study the adiabatic evolution of the instantaneous eigenstates of a two-level quantum hamiltonian with a adiabatically-varying periodic external classical electric field, in the electric dipole approximation. In section II we calculate explicitly the geometric phases of this model from the expression given by Berry[4], and explore some consequences for real and complex effective classical electric fields. In section III, for the sake of verification, we map this model onto the spin- $1 / 2$ model under the action of an external classical magnetic field to discuss the presence/absence of Berry's phase in the instantaneous eigenstates of the two-level model for a time-dependent classical electric field, including the RWA of the model. Finally, in section IV we summarize our results for two-level hamiltonian with an arbitrary periodic time-dependent external electric field.

\section{THE INTERACTING TWO-LEVEL MODEL}

The hamiltonian $\mathbf{H}_{0}$ of the quantum two-level model, without interaction, written in terms of its eigenstates is

$$
\mathbf{H}_{0}=\varepsilon_{1}|1\rangle\left\langle 1\left|+\varepsilon_{2}\right| 2\right\rangle\langle 2|,
$$

where $\mathbf{H}_{0}|i\rangle=\varepsilon_{i}|i\rangle, i=1,2$. We consider $\varepsilon_{1}<\varepsilon_{2}$, which prevents (1) from describing a degenerate two-level system.

A first motivation to study a two-level model comes from an original quantum system confined to a spatial region of 
finite diameter, interacting with a classical time-dependent external electric field $\vec{E}(t)$, the wavelength of which being much larger than the diameter of the region and in the quasiresonant regime. This last two conditions allow us to use the electric dipole approximation for the interaction in the RWA. The interaction hamiltonian $\mathbf{H}_{e}(t)$, represented in the basis of the eigenstates of $\mathbf{H}_{0}$, is [14]

$$
\mathbf{H}_{e}(t)=\left(\begin{array}{cc}
\varepsilon_{1} & \left(\vec{d}_{12} \cdot \vec{E}(t)\right)^{*} \\
\vec{d}_{12} \cdot \vec{E}(t) & \varepsilon_{2}
\end{array}\right),
$$

in which

$$
\vec{d}_{12} \equiv-e\langle 1|\overrightarrow{\mathbf{x}}| 2\rangle
$$

and $\overrightarrow{\mathbf{x}}$ is the position operator of the electron. We assume that the eletronic states $|i\rangle, i=1,2$, are spherically symmetric, and hence do not have a permanent electric dipole $\left(\vec{d}_{i i}=0\right.$, $i=1,2$ ). We are using the natural units, $\hbar=c=1$.

The components of the vector $\vec{d}_{12}$ can be imaginary numbers, depending on the choice of the basis $\{|i\rangle, i=1,2\}$ and they are time-independent. Throughout our calculations, we have chosen the basis of eigenstates of $\mathbf{H}_{0}$, such that the components of $\vec{d}_{12}$ are real; we have taken $e>0$, so that the electronic charge is $-e$.

The RWA of hamiltonian (2) has been used in quantum computation to implement geometric phase gates between ion-qubits in the presence of an external electric field, whose wavelength are much larger than the space distribution of the atom and it is in the quasi-resonant regime[12, 15-17]. The geometric operations associated to the time evolution of hamiltonian (2) are considered to be potentially robust[15].

For the sake of future comparison between the adiabatic evolution of the two-level hamiltonian (2) and that of another two-level model that describes the interaction of the spin-1/2 with an external magnetic field[4], we make a trivial shift in the hamiltonian and define

$$
\mathbf{H}_{e}^{\prime}(t) \equiv \mathbf{H}_{e}(t)-\frac{\left(\varepsilon_{1}+\varepsilon_{2}\right)}{2} \mathbf{1},
$$

in which $\mathbf{1}$ is the $2 \times 2$ identity matrix. In order to simplify the mathematical expressions in $\mathbf{H}_{e}^{\prime}(t)$ we define

$$
\begin{gathered}
\Delta \varepsilon \equiv \varepsilon_{2}-\varepsilon_{1}, \\
D(t) \equiv \vec{d}_{12} \cdot \vec{E}(t) .
\end{gathered}
$$

We finally have

$$
\mathbf{H}_{e}^{\prime}(t)=\left(\begin{array}{cc}
-\frac{\Delta \varepsilon}{2} & (D(t))^{*} \\
D(t) & \frac{\Delta \varepsilon}{2}
\end{array}\right) .
$$

From now on, we study the time evolution of the quantum system driven by a general hamiltonian of the type (6) when it evolves adiabatically. We have $\Delta \varepsilon \in \mathbb{R}$ and $D(t) \in \mathbb{C}$; the latter function has a period $T$ satisfying the adiabatic condition $T \cdot\left(\frac{\Delta \varepsilon}{2 \pi}\right) \gg 1$. Any function $D(t)$ can be written in the form (5b); the effective external electric field $\vec{E}(t)$ evolves adiabatically and is out of the quasi-resonant regime. In order to have a slow-varying periodic function $D(t)$, it is sufficient that the effective external field have an adiabatically-evolving periodic component along the direction of the time-independent vector $\vec{d}_{12}$. We continue to assume that the hamiltonian (6) is non-degenerate at any instant $t$.

Following Berry's original notation [4], in the general case we have a hamiltonian that varies in time due to a timedependent classical field $\vec{R}(t)$, that is, $\mathbf{H}(t)=\mathbf{H}(\vec{R}(t))$. The classical field $\vec{R}(t)$ is supposed to evolve adiabatically. The instantaneous eigenvalue equation of energy is

$$
\mathbf{H}(\vec{R}(t))|n ; \vec{R}(t)\rangle=\lambda_{n}(\vec{R}(t))|n ; \vec{R}(t)\rangle,
$$

where $|n ; \vec{R}(t)\rangle$ is the instantaneous eigenvector of hamiltonian $\mathbf{H}(\vec{R}(t))$ with eigenvalue $\lambda_{n}(\vec{R}(t))$. The instantaneous spectrum $\left\{\lambda_{n}(\vec{R}(t))\right\}$ is supposed to be non-degenerate. From Ref.[4], each eigenstate $|n ; \vec{R}(t)\rangle$ acquires the following Berry's phase:

$$
\gamma_{n}=-i \oint_{\Gamma_{\vec{R}}}\langle n ; \vec{R}(t)| \vec{\nabla}_{\vec{R}}(|n ; \vec{R}(t)\rangle) \cdot d \vec{R},
$$

in which $\Gamma_{\vec{R}}$ is an oriented closed loop in the $\vec{R}$-parameter space.

In the two-level hamiltonian (6), coupled to an effective classical electric field, [cf. eqs.(2)-(6)] we have $\vec{R}(t)=\vec{E}(t)$. In order to include the RWA of this hamiltonian, we consider the general case where the components of the effective classical field, vector $\vec{E}(t)$, can be complex.

From now on, we have

$$
\mathbf{H}(\vec{R}(t))=\mathbf{H}_{e}^{\prime}(\vec{E}(t)) .
$$

The time dependence of the hamiltonian $\mathbf{H}_{e}^{\prime}$, of the function $D$ and of the eigenvalues $\lambda_{n}$ is solely due to the time dependence of the classical electric field. In order to simplify the notation, we write this operator and functions as $\mathbf{H}_{e}^{\prime}(t), D(t)$ and $\lambda_{n}(t)$, respectively.

The hamiltonian $\mathbf{H}_{e}^{\prime}(t)$ has two instantaneous eigenvalues:

$$
\lambda_{l}(t)=l \sqrt{\left(\frac{\Delta \varepsilon}{2}\right)^{2}+|D(t)|^{2}}, \quad l= \pm 1 .
$$

The instantaneous normalized eigenstates of $\mathbf{H}_{e}^{\prime}(t)$, associated to $\lambda_{l}(t)$, for $l= \pm 1$, are

$$
W_{l}(t)=\left(\begin{array}{c}
\left(A_{l}(t)\right)^{1 / 2} \\
\frac{l}{\sqrt{2}} D(t)\left(F_{l}(t)\right)^{-1 / 2}
\end{array}\right)
$$

$$
\equiv\left(\begin{array}{c}
u_{l}(t) \\
v_{l}(t)
\end{array}\right)
$$

so that

$$
A_{l}(t) \equiv \frac{1}{2}-l \cdot \frac{\Delta \varepsilon}{4} \frac{1}{\sqrt{\left(\frac{\Delta \varepsilon}{2}\right)^{2}+|D(t)|^{2}}}
$$

and

$$
F_{l}(t)=\left(\frac{\Delta \varepsilon}{2}\right)^{2}+|D(t)|^{2}-l \cdot\left(\frac{\Delta \varepsilon}{2}\right) \sqrt{\left(\frac{\Delta \varepsilon}{2}\right)^{2}+|D(t)|^{2}} .
$$


Using the entries of $W_{l}(t)$, after a closed loop in the $\vec{E}$ parameter space, we can write Berry's phase $\gamma_{l}$ (see eq. (8)) as

$$
\gamma_{l}=-i \oint_{\Gamma_{\vec{E}}}\left[u_{l}^{*}\left(\vec{\nabla}_{\vec{E}} u_{l}\right)+v_{l}^{*}\left(\vec{\nabla}_{\vec{E}} v_{l}\right)\right] \cdot d \vec{E}, \quad \text { for } \quad l= \pm 1 .
$$

We now calculate $\gamma_{+1}$ explicitly. Since

$$
\frac{\partial u_{+1}}{\partial E_{i}}=\frac{1}{2} \frac{1}{\left(A_{+1}\right)^{1 / 2}} \frac{\partial A_{+1}}{\partial E_{i}}, \quad i \in\{x, y, z\},
$$

we obtain

$$
u_{+1}^{*}\left(\vec{\nabla}_{\vec{E}} u_{+1}\right)=\vec{\nabla}_{\vec{E}}\left[\frac{A_{+1}(t)}{2}\right]
$$

that is a gradient in $\vec{E}$-parameter space.

In the general case (including the RWA of hamiltonian $\left.\mathbf{H}_{e}^{\prime}(t)\right)$ we have that $D(t) \in \mathbb{C}$, then

$$
D(t)=|D(t)| e^{i \phi(t)},
$$

where $\phi(t)$ is a real phase. Substituting eq.(17) in $v_{+1}(t)$ yields

$$
v_{+1}(t)=\frac{|D(t)|}{\sqrt{2}} \frac{e^{i \phi(t)}}{\left(F_{+1}(t)\right)^{1 / 2}} .
$$

The derivative of $v_{+1}(t)$ with respect to the components of the external electric field $\vec{E}$ is

$$
\begin{aligned}
& \frac{\partial v_{+1}}{\partial E_{i}}=\frac{e^{i \phi(t)}}{\sqrt{2}}\left[\frac{1}{F_{+1}^{1 / 2}} \frac{\partial[|D(t)|]}{\partial E_{i}}\right. \\
& \left.-\frac{|D(t)|}{2 F_{+1}^{3 / 2}} \frac{\partial\left[F_{+1}(t)\right]}{\partial E_{i}}+\frac{i|D(t)|}{F_{+1}^{1 / 2}} \frac{\partial[\phi(t)]}{\partial E_{i}}\right],
\end{aligned}
$$

in which $i \in\{x, y, z\}$. Remembering that $v_{+1}(t) \in \mathbb{C}$, we have

$$
v_{+1}^{*}\left(\vec{\nabla}_{\vec{E}} v_{+1}\right)=\vec{\nabla}_{\vec{E}}\left[\frac{|D(T)|^{2}}{4 F_{+1}(t)}\right]+\frac{i}{2} \frac{|D(T)|^{2}}{F_{+1}(t)}\left[\vec{\nabla}_{\vec{E}}(\phi(t))\right] .
$$

Substituting results (16) and (20) on the r.h.s. of eq. (14) for $l=+1$, it becomes

$$
\begin{aligned}
& \gamma_{+1}=-i \oint_{\Gamma_{\vec{E}}}\left[\vec{\nabla}_{\vec{E}}\left[\frac{A_{+1}(t)}{2}+\frac{|D(T)|^{2}}{4 F_{+1}(t)}\right]\right. \\
& \left.+\frac{i}{2} \frac{|D(T)|^{2}}{F_{+1}(t)}\left[\vec{\nabla}_{\vec{E}}(\phi(t))\right]\right] \cdot d \vec{E} .
\end{aligned}
$$

The integral of the first term on the r.h.s. of expression (21) vanishes for a closed loop $\Gamma_{\vec{E}}$. Rewriting the second term on the r.h.s. of this expression, we obtain

$$
\gamma_{+1}=\frac{1}{2} \int_{0}^{T} d t \frac{|D(t)|^{2}}{F_{+1}(t)} \dot{\phi}(t)
$$

where $T$ is the period of the function $D(t)(D(0)=D(T))$ and $\dot{\phi}(t) \equiv \frac{d \phi(t)}{d t}$. $\pm 1$

The calculation of $\gamma_{-1}$ is analagous and we obtain, for $l=$

$$
\gamma_{l}=\frac{l}{2} \int_{0}^{T} d t \frac{|D(t)|^{2}}{F_{l}(t)} \dot{\phi}(t)
$$

A few comments on the geometric phase of the instantaneous eigenstates of $\mathbf{H}_{e}^{\prime}(t)$ (see hamiltonian (6), or (2) when it is written in terms of an effective classical electric field, are in order.

i) From the result (16), we verify that the entry $u_{l}(t)$ in the eigencolumns of $W_{l}(t)$, for $l= \pm 1$, does not contribute to the geometric phase $\gamma_{l}$.

ii) For $D(t) \in \mathbb{R}$ (or, equivalently, $\vec{E}(t) \in \mathbb{R}^{3}$ ) the phase $\phi(t)$ is time-independent, and $\mathbf{H}_{e}^{\prime}(t)$ is a one-parameter hamiltonian. Therefore its instantaneous eigenstates do not acquire a geometric phase along a closed loop in the $\vec{E}(t)$-space. This last statement agrees with the result derived from eq. (23) for real $D(t)$.

iii) For $D(t) \in \mathbb{C}$ we can have a non-null geometric phase $\gamma_{l}, l= \pm 1$, since $\mathbf{H}_{e}^{\prime}(t)$ becomes a two-parameter hamiltonian. This case corresponds to the RWA of $\mathbf{H}_{e}^{\prime}(t)$ when we have $\vec{E}(t) \in \mathbb{C}^{3}$.

iv) Two complex fields $\vec{E}_{1}(t)$ and $\vec{E}_{2}(t)$ with the same component along the vector $\vec{d}_{12}(t)$ have the same geometric phase $\gamma_{l}, l= \pm 1$.

v) For complex fields $\vec{E}(t)$ it suffices to have a periodic component of this field along $\vec{d}_{12}(t)$ for the instantaneous eigenstates of $\mathbf{H}_{e}^{\prime}(t)$ to acquire a geometric phase.

vi) For a complex monochromatic classical electric field,

$$
\vec{E}_{ \pm}(t)=\vec{E}_{0} e^{\mp i\left(\omega t+\phi_{0}\right)}
$$

in which $\omega$ is the angular frequency of the classical field, $\vec{E}_{0}$ is its real amplitude and $\phi_{0}$ is a real constant phase. From expression (23) we obtain the relation between the geometric phases $\gamma_{l}^{(+)}$and $\gamma_{l}^{(-)}$, respectively related to the monochromatic electric fields $\vec{E}_{+}(t)$ and $\vec{E}_{-}(t)$, which reads

$$
\gamma_{l}^{(+)}=-\gamma_{l}^{(-)}, \quad \text { for } l= \pm 1 .
$$

Relation (25) comes from the linear dependence on $D(t)$ in the numerator of the entry $v_{l}(t)$ (see eq. (11a)). As a consequence of this result we obtain that for a hamiltonian (2) in the presence of a classical field of the form $\vec{E}(t)=\vec{E}_{+}(t)+\vec{E}_{-}(t)$, its instantaneous eigenstates do not acquire a geometric phase.

vii) Finally, we verify the absence of Berry's phase in a two-level quantum system coupled to any real classical external electric field, in the electric dipole approximation and in the adiabatic regime. This result comes from the fact that for this hamiltonian (2) we have 
$\left\langle n ; \vec{R}(t)\left|\vec{\nabla}_{\vec{R}}\right|(|n ; \vec{R}(t)\rangle)=\vec{\nabla}_{\vec{E}}[G(\vec{E})]\right.$, that corresponds to a scalar potencial $G(\vec{E})$ of a conservative system for any time-dependent real classical electric field, thus yielding null integrals for closed paths in $\vec{E}$-parameter space.

\section{COMPARISON OF TWO-LEVEL MODELS}

In his seminal paper[4], Berry showed that geometric phases are acquired by the instantaneous eigenstates of a spin$1 / 2$ particle under a precessing magnetic field $\vec{B}(t)$ in adiabatic evolution, described by the hamiltonian

$$
\mathbf{H}_{\text {spin }}(t)=\mu \vec{\sigma} \cdot \vec{B}(t)
$$

in which $\mu=\frac{1}{2} g \mu_{B}, g$ is the Landé factor and $\mu_{B}$ is the Bohr magneton.

In order to compare the adiabatic evolution of the two-level models $\mathbf{H}_{e}^{\prime}(t)$ (see eq. (4)) and $\mathbf{H}_{\text {spin }}(t)$ (eq. (26)) we use the Pauli matrices to rewrite hamiltonian (4) as

$$
\mathbf{H}_{e}^{\prime}(t)=\Re\left[\vec{d}_{12} \cdot \vec{E}(t)\right] \sigma_{x}+\mathfrak{I}\left[\vec{d}_{12} \cdot \vec{E}(t)\right] \sigma_{y}+\frac{\left(\varepsilon_{1}-\varepsilon_{2}\right)}{2} \sigma_{z},
$$

in which $\mathfrak{R}[\ldots]$ and $\mathfrak{I}[\ldots]$ stand for the real and imaginary parts of the expression in brackets, respectively.

Comparing hamiltonians (26) and (27), we obtain that $\mathbf{H}_{e}^{\prime}(t)$ is equivalent to the spin hamiltonian in the presence of an external classical magnetic field with components

$$
\begin{aligned}
B_{x}(t) & \equiv \frac{1}{\mu} \Re\left[\vec{d}_{12} \cdot \vec{E}(t)\right], \\
B_{y}(t) & \equiv \frac{1}{\mu} \mathfrak{I}\left[\vec{d}_{12} \cdot \vec{E}(t)\right], \\
B_{z}(t) & \equiv \frac{\varepsilon_{1}-\varepsilon_{2}}{2 \mu} .
\end{aligned}
$$

In the general case, we use the definition (5b) to rewrite the scalar product

$$
\begin{aligned}
\vec{d}_{12} \cdot \vec{E}(t) & \equiv D(t) \\
& \equiv|D(t)| e^{i \phi(t)} .
\end{aligned}
$$

Substituting expression (29b) in the components of the equivalent magnetic field, we obtain

$$
\begin{aligned}
& B_{x}(t)=\frac{|D(t)|}{\mu} \cos [\phi(t)], \\
& B_{y}(t)=\frac{|D(t)|}{\mu} \sin [\phi(t)], \\
& B_{z}(t)=\frac{\varepsilon_{1}-\varepsilon_{2}}{2 \mu} .
\end{aligned}
$$

These equations describe an external magnetic field with a constant $z$-component. For any real vector $\vec{E}(t)$ we have $B_{y}(t)=0$ and the equivalent magnetic field varies along a straight line in the $(x, z)$ plane. The path being a straight line in the parameter space of the magnetic field yields a null solid angle, and hence produces no geometric phase to the instantaneous eigenstates of $\mathbf{H}_{e}(t)$ [4]. Such absence of a geometric phase happens for a quantum system under a classical external eletric field $\vec{E}$ that follows a closed loop in its parameter space. This result is valid for any periodic classical electric field with real components.

Although the hamiltonian of a two-level model must be hermitian, we have an important application of this model in quantum optics, namely for a monochromatic time-dependent external electric field $\vec{E}(t)$ in the quasi-resonant regime[3]. Under these conditions the dynamics of the electronic part of the quantum model is well approximated by the RWA of the hamiltonian (2). In this approximation, the main contribution to the transition probability amplitude comes from the positive frequency of $\vec{E}_{+}(t)$ (see eq. (24)). in this case, he components of the effective magnetic field a time dependent phase $\phi(t)$ given by

$$
\phi(t) \equiv-\left(\omega t+\phi_{0}\right) .
$$

The previous magnetic field configuration describes a precessing field with a non-null solid angle. The instantaneous eigenstates of the RWA of the two-level model acquire a geometric phase under an adiabatic periodic evolution of the monochromatic electric field (24), which is already known in the literature[8].

The results of section III fully agree with the consequences of expression (23) for Berry's phases $\gamma_{l}, l= \pm 1$, associated to the instantaneous eigenstates of hamiltonian $\mathbf{H}_{e}^{\prime}$.

\section{CONCLUSIONS}

We study the adiabatic evolution of a two-level quantum model driven by hamiltonians of type (6), which appears in the interaction of matter with a classical electric field in the quasi-resonant regime[3]. The interaction is taken in the electric dipole approximation and the hamiltonian is handled in RWA. The RWA of hamiltonian (2) has been used in the modelling of prototype systems for qubits in quantum computation, constituted by atoms interacting with electric fields[12].

By explicitly calculating Berry's phases of the instantaneous eigenstates of hamiltonian $\mathbf{H}_{e}^{\prime}(t)$, see eq. (4), we show that they are absent when the quantum system is under the action of any time-dependent real classic electric field that evolves adiabatically. This result is a direct consequence of the quantum system being driven by a one-parameter hamiltonian. For any real time-dependent electric field, the geometric phase is calculated over a closed loop of a gauge field with a conservative potential, thus giving a null result. Certainly, this result is unexpected since it is valid for any real time-dependent electric field, which in general is a nonconservative field.

On the other hand, we can have geometric phases acquired by the instantaneous eigenstates of the RWA of hamiltonian 
$\mathbf{H}_{e}^{\prime}(t)$; a sufficient condition is that the component of the classical electric field along the direction of $\vec{d}_{12}$, defined by expression (3), be periodic and evolve adiabatically.

A very interesting result comes from the $\phi$-dependence of Berry's phase (eq. (23)). Interactions with the positivefrequency part $\vec{E}_{+}(t)$ and negative-frequency part $\vec{E}_{-}(t)$ of a real monochromatic electric field yield contributions to the geometric phase that differ only by a global sign. If the quantum system interacts with the (real) superposition of these two components of $\vec{E}$ the geometric phases of the instantaneous eigenstates of the interaction hamiltonian vanish. This set of consequences derived from eq. (23) rise the question whether the two-level model (2) is suitable for the calculation of geometric phases in the interaction of atoms with monochromatic laser fields, in the quasi-resonant regime[12, 15-17] or whether the original hamiltonian for the atom with an infinite number of bound states in the presence of a real monochromatic electric field should be used instead. In order to verify the correction of the results of section II, hamiltonian
(2) is mapped onto a spin-1/2 model coupled to an external magnetic field[8]. The presence of geometric phases in this last model was showed by Berry in his seminal paper[4]. In Ref.[8] Garrison and Wright showed that the instantaneous eigenstates of the RWA of the two-level model in the presence of a monochromatic classical electric field acquire geometric phases.

The authors are in debt with Sir Michael V. Berry for his comments on the original version of the present communication. He raised the question of the validity of using the RWA of hamiltonian (2) to calculate the geometric phases of atoms in the presence of a monochromaticelectric field in the quasiresonant regime.

M.T. Thomaz (Fellowship CNPq, Brazil, Proc.No.: 30.0549/83-FA) thanks CNPq for partial financial support.
[1] I.I. Rabi, Phys. Rev. 49, 324-328 (1936).

[2] I.I. Rabi, Phys. Rev. 51, 652-654 (1937).

[3] P. Meystre and Murray Sargent III, "Elements of Quantum Optics", $3^{\text {rd }}$ ed., Springer- Verlag (1998), section 3.3.

[4] M. V. Berry, Proc. R. Soc. London, Ser. A 392, 45-57 (1984).

[5] A.C. Aguiar Pinto, M.C. Nemes, J.G. Peixoto de Faria and M.T. Thomaz, Am. J. Phys. 68, 955-958 (2000).

[6] M. Born and V. Fock, Z. Phys. 51, 165-180 (1928).

[7] M. A. Alves and M. T. Thomaz, Am. J. Phys. 75, 552-560 (2007).

[8] J.C. Garrison and E.M. Wright, Phys. Lett. A128, 177-181 (1988).

[9] S.P. Tewari, Phys. Rev. A39, 6082-6085 (1989).
[10] A. Kvitsinsky and S. Putterman, J. Math. Phys. 32, 1403-1407 (1990).

[11] Y. Kanayanuma, Phys. Rev. A55, R2495-R2498 (1997).

[12] D. Leibfried et al., Nature (London)422, 412- 415 (2003).

[13] Y. Aharonov and J. Anandan, Phys. Rev. Lett. 58, 1593-1596 (1987).

[14] W. E. Lamb Jr., R.R. Schicher and M.O. Scully, Phys. Rev. A36, 2763-2772 (1987).

[15] M. Tian et al., Phys. Rev. A69, 050301-1-4 (2004).

[16] H. Imai, Y. Otsubo and A. Morinaga, Phys. Rev. A76, 0121161-4 (2007)

[17] H. Imai and A. Morinaga, Phys. Rev. A76, 062111-1-4 (2007). 